\title{
Design and Calibration of a mm-Wave Personal Exposure Meter for 5G Exposure Assessment in Indoor Diffuse Environments
}

\author{
Reza Aminzadeh ${ }^{1}$. \\ Abdou Khadir Fall ${ }^{2}$. Jérôme Sol $^{2}$. \\ Arno Thielens ${ }^{1,3}$. Philippe Besnier ${ }^{2}$. \\ Maxim Zhadobov $^{4}$. Nele De Geerter ${ }^{5}$. \\ Prakash Parappurath Vasudevan ${ }^{1,6}$. \\ Luc Dupré ${ }^{5}$. Roel Van Holen ${ }^{6}$. \\ Luc Martens $^{1}$. Wout Joseph ${ }^{1}$
}

Received: date / Accepted: date

\begin{abstract}
For the first time, a mm-wave personal exposure meter (mm-PEM) for the 5th generation of mobile networks (5G) exposure assessment in indoor diffuse fields is presented. The design is based on simulations and onphantom calibration measurements in a mm-wave reverberation chamber (RC) at $60 \mathrm{GHz}$. The mm-PEM consists of an array of nine antennas on the body. Using the mm-PEM, the incident power density (IPD) is measured in the unloaded RC, for the antenna(s) on the phantom and RC loaded with phantom. The uncertainty of the mm-PEM is then determined in terms of its response, which is defined as the ratio of antenna aperture for the above measurement scenarios. Using nine antennas, the designed meter has a response of 1.043 $(0.17 \mathrm{~dB})$ at $60 \mathrm{GHz}$ which is very close to $1(0 \mathrm{~dB})$, the desired ideal response value. The mm-PEM measured an IPD of $96.6 \mathrm{~W} . \mathrm{m}^{-2}$ at $60 \mathrm{GHz}$ in the RC, for an input power of $1 \mathrm{~W}$. In addition, the average absorption cross section of the phantom is determined as $225 \mathrm{~cm}^{2}$, which is an excellent agreement with its physical dimensions.
\end{abstract}

Reza Aminzadeh

E-mail: reza.aminzadeh@ugent.be

${ }^{1}$ Department of Information Technology, Ghent University/imec, B-9052 Ghent, Belgium

2 Institute of Electronics and Telecommunications of Rennes, UMR CNRS 6164, INSA of Rennes, Rennes 35708, France

3 Berkeley Wireless Research Center, Department of Electrical Engineering and Computer Sciences, University of California, Berkeley, 2108 Allston Way, Suite 200, Berkeley, CA 94704, United States

${ }^{4}$ Institute of Electronics and Telecommunications of Rennes, UMR CNRS 6164, University of Rennes 1, Rennes 35042, France

5 Dept. of Electrical Energy, Systems and Automation (EESA), Ghent, Belgium

6 Dept. of Electronics and Information Systems (Elis), Ghent University/imec, Ghent, Belgium 
Keywords mm-wave personal exposure meter $\cdot 5 \mathrm{G} \cdot$ diffuse fields $\cdot$ indoor environments $\cdot$ exposure $\cdot$ average absorption cross section

\section{Introduction}

The Fifth generation of mobile networks (5G) is expected to become commercially available by 2020 [1]. Current research activities on $5 \mathrm{G}[1,2]$ involve various topics including large data rates (up to 10 Gbps [1]), reliability, and security, among others. 5G technology will mainly deploy the available spectrum above $10 \mathrm{GHz}$ including the mm-wave band $[1,3,4]$ to facilitate such high speed data transmission. Once this technology is commercially available, the general population will be exposed to EM fields at these frequencies. Therefore, it becomes crucial to measure the personal exposure to electromagnetic fields (EMFs) in the mm-waves band. Any wireless device has to comply with exposure limits such as those issued by the International Commission on NonIonizing Radiation Protection (ICNIRP) [5]. According to the ICNIRP, the incident power density (IPD) is used as a dosimetric quantity. The IPD is limited to $1 \mathrm{~mW} / \mathrm{cm}^{2}$ and $5 \mathrm{~mW} / \mathrm{cm}^{2}$ for general public and occupational exposure, respectively.

A typical approach to measure the personal exposure to RF EMFs is to use personal exposure meters (PEMs) [6]. PEMs are body-worn devices that are equipped with an antenna and receiver electronics to register the EMFs on body. Research has shown that PEMs may underestimate the EMFs due to the presence (shadowing) of the body [6-9]. A review of different sources of PEMs' uncertainties can be found in [10]. Wearable personal distributed exposure meters (PDEs) have been demonstrated for single [8] and multi telecommunication bands [11]. A PDE consists of multiple antennas on the body. The use of multiple locations reduces the variations on the measurements and thus the uncertainty. The necessity of using multiple locations for mm-wave personal exposure assessment is shown in [12]. A similar approach is used in [13] for the $60 \mathrm{GHz}$ band. Using three antennas, the $60 \mathrm{GHz}$ exposure meter [13] has been calibrated on the arm of a human subject in anechoic conditions.

Current research on the 5G exposure is mainly focused on assessment of human exposure to mm-wave antennas used for user devices and portable equipments. An example is [14] in which the human exposure to phased arrays is studied in the range of 10-60 GHz. similarly, [15] studied human body exposure to a $5 \mathrm{G}$ mobile terminal operating at $\mathrm{mmW}$. The previous dosimetric research at mm-waves have been mainly focused on animal $[16]$, cells $[17,18]$ and human skin [19-21] exposure.

On the one hand, high propagation loss and low building penetration at $60 \mathrm{GHz}$, makes mm-waves suitable for low range indoor but high speed communications [4]. Consequently, a high isolation between outdoor and indoor networks exists. On the other hand, research shows that people spend most (more than $80 \%$ ) of their time indoors [22]. This could increase the exposure to EMFs at mm-waves in indoor environments. The total power in an indoor 
environment consists of specular and dense (or diffuse) multipath components (DMC) [23]. The former is the result of coherent reflections from large surfaces i.e., walls. The latter is the reason of non-coherent reflections, due to the presence of different objects in an indoor area. The contribution of the DMC to the power density in an indoor area may increase up to $95 \%$ [24] compared to outdoor areas.

The novelties of this research are as follows. The goal is to, for the first time, design and calibrate a personal exposure meter (mm-PEM) for RF exposure assessment at $60 \mathrm{GHz}$ in indoor (diffuse) environments. The mm-PEM is designed based on calibrations in a mm-wave reverberation chamber ( $\mathrm{RC}$ ). The design of the mm-wave $\mathrm{RC}$ is presented in $[25,26]$. The goal of calibrations is as follows: First, to find the configuration for the nodes on body (location and orientation); Second, to measure the actual IPD in free space in diffuse fields and correct the measurements for the presence of the human body. In addition, the IPD in a mm-wave RC is measured using an isotropic tri-axial probe. Finally, the uncertainty of the proposed mm-PEM consisting of an array of nine antennas is determined.

\section{Materials and Methods}

The mm-PEM is calibrated in a mm-wave RC in the frequency range of 59.5 to $60.5 \mathrm{GHz}$. The antenna used for the mm-PEM is presented in Section 2.1. A horn antenna (Section 2.2) and a broadband isotropic probe (Section 2.3) are used as reference to measure the IPD in the RC (Section 2.4). These reference values are then compared with the measured values with the mmPEM. The measurement uncertainty of the mm-PEM is studied experimentally (Section 2.5) and numerically (Section 2.6).

\subsection{Single antenna for mm-PEM}

The $60 \mathrm{GHz}$ antenna used in this study is a microstrip four-patch single layer antenna array (denoted as Rx in the text) [27] and is shown in Fig. 1. This four-patch antenna will be used as an individual element of the nine-element array configuration resulting in the mm-PEM in Section 2.5 (see Fig. 5(a)). The antenna is printed on a thin $(127 \mu \mathrm{m})$ RT Duroid 5880 substrate. At $60 \mathrm{GHz}$, the $\mathrm{Rx}$ has a gain of $11.8 \mathrm{dBi}$, directivity of $13.9 \mathrm{dBi}$ and a total radiation efficiency of $60 \%$ in free space.

\subsection{Horn antenna}

A standard gain V-band horn antenna is used to measure the IPD (denoted as $S_{i n c}$ in the text) in the empty RC. The antenna has a gain of $20 \mathrm{dBi}$. The measured values using the horn antenna are used as a reference to be compared to the measurements of the mm-PEM in the unloaded (empty) RC. 


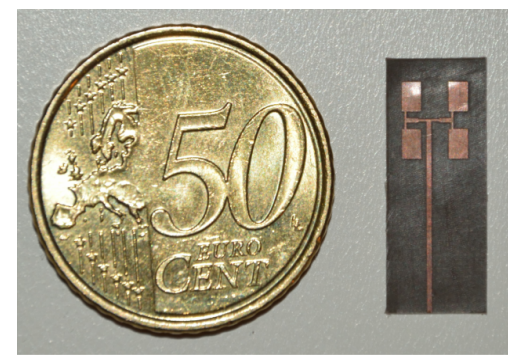

Fig. 1 The $60 \mathrm{GHz}$ four-patch antenna array that is used for the mm-PEM and is denoted as Rx in the text. The antenna is fabricated on an RT Duroid 5880 substrate.

\subsection{Broadband isotropic field meter}

An isotropic E-field probe (EF6092, Narda, Hauppauge, NY, USA) is used with a broadband field meter (NBM 550, Narda, Hauppauge, NY, USA) to measure $S_{i n c}$ in the empty RC. The frequency range of the probe (EF6092) is $100 \mathrm{MHz}$ to $60 \mathrm{GHz}$. This probe has isotropic tri-axial sensors that measure the E-field components in the RC. The measured $S_{\text {inc }}$ could be used as a reference for calibration of the mm-PEM.

\subsection{Reverberation chamber}

The RC is made of aluminum walls with a thickness of $10 \mathrm{~mm}$ (Fig. 3). The inner dimensions of the $\mathrm{RC}$ are $0.58 \times 0.592 \times 0.595 \mathrm{~m}^{3}$. A metallic mode stirrer and a DC motor continuously rotate to generate random fields in the $\mathrm{RC}$. The $\mathrm{RC}$ operates in the 58.5-61.5 GHz range and has a measured Q-factor in the order of $10^{4}$ to $10^{5}$. A WR-15 open-ended waveguide is used as a transmitter $(\mathrm{Tx})$; it is fed by either a signal generator or a vector network analyzer.

\subsection{Calibration Measurement Setup}

Figure 2 shows the diagram of the proposed setup for the calibration measurements to design an array resulting in the mm-PEM. Calibrations are performed for two scenarios: empty RC and loaded RC.

Setup 1 (see Section 2.5.1) is proposed to measure the IPD in the empty $\mathrm{RC}$ using a broadband isotropic field probe and a standard gain horn antenna as a reference.

The aim of Setup 2 (see Section 2.5.2) is to determine a response for the proposed mm-PEM. The S-parameters are used to calculate the IPD and response of the mm-PEM. The latter is defined as the ratio of the antenna apertures (AA)s for the above-mentioned scenarios.

Finally, the average absorption cross section (AACS) of the phantom is calculated from the measured Q-factors of the RC using the mm-PEM. 


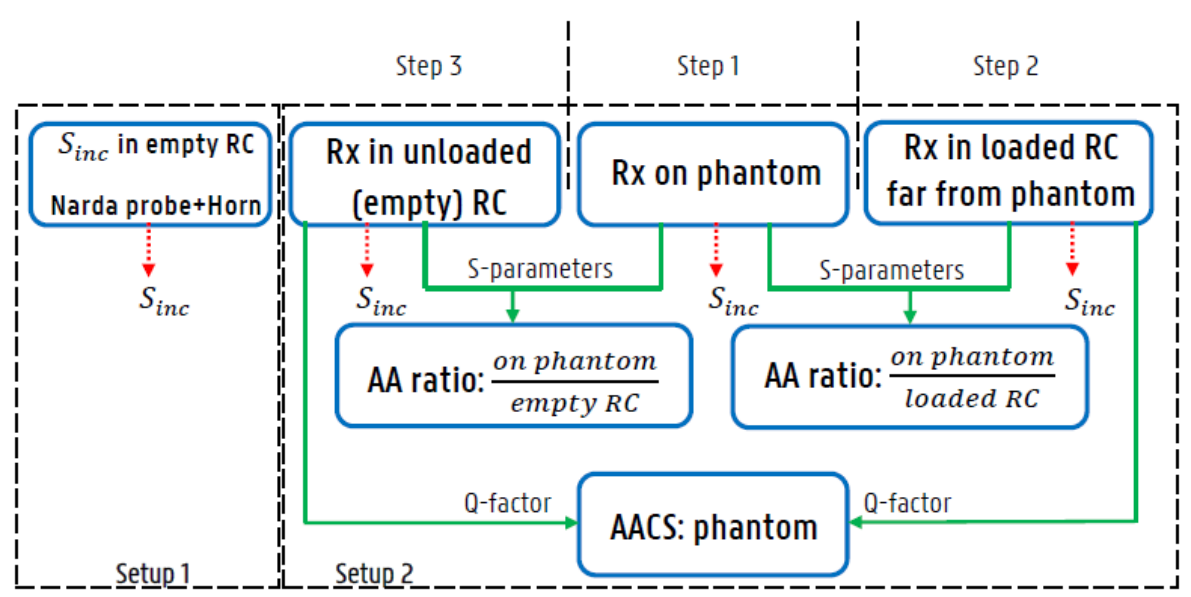

Fig. 2 The diagram of the proposed setup for calibration measurements. $S_{\text {inc }}$ : incident power density. For setup 2, the values are averaged over nine antennas. AA: antenna aperture; AACS: average absorption cross section.

\subsubsection{Setup 1: Measurements using broadband field meter}

The goal is to measure the IPD in the empty RC. Figure 3 depicts the proposed measurement setup in the empty RC. A mm-wave source (Quinstar Technology Inc, Torrance, CA, USA) generates EM fields in the range of $58.3-61.6 \mathrm{GHz}$ and an open-ended waveguide radiates the generated fields inside the RC. During a full rotation, the stirrer goes through 100 positions. A broadband isotropic probe (EF6092) is placed in the center of RC and is connected to a field meter (NBM550) located outside the RC measuring the IPD at $60 \mathrm{GHz}$ and a bandwidth of $3.2 \mathrm{GHz}$. The EF6092 probe has been calibrated by the manufacturer prior to the measurements. Additionally, a receiving V-band horn antenna connected to an E4418B (Agilent, Santa Clara, CA, USA) power meter is used to measure the power in the empty $\mathrm{RC}$. The received power density on the horn antenna $S_{i n c, h o r n}$ is calculated as:

$$
S_{\text {inc,horn }}=\frac{P_{r, h o r n}}{A A_{\text {horn }}}
$$

where $P_{r, h o r n}$ is the recorded power received by the horn antenna and $A A_{\text {horn }}$ is the effective area of the horn antenna inside the RC [28]:

$$
A A_{h o r n}=\frac{\eta \lambda^{2}}{8 \pi}
$$

where $\eta$ is radiation efficiency of the horn antenna (0.94) and $\lambda$ is the wavelength. 

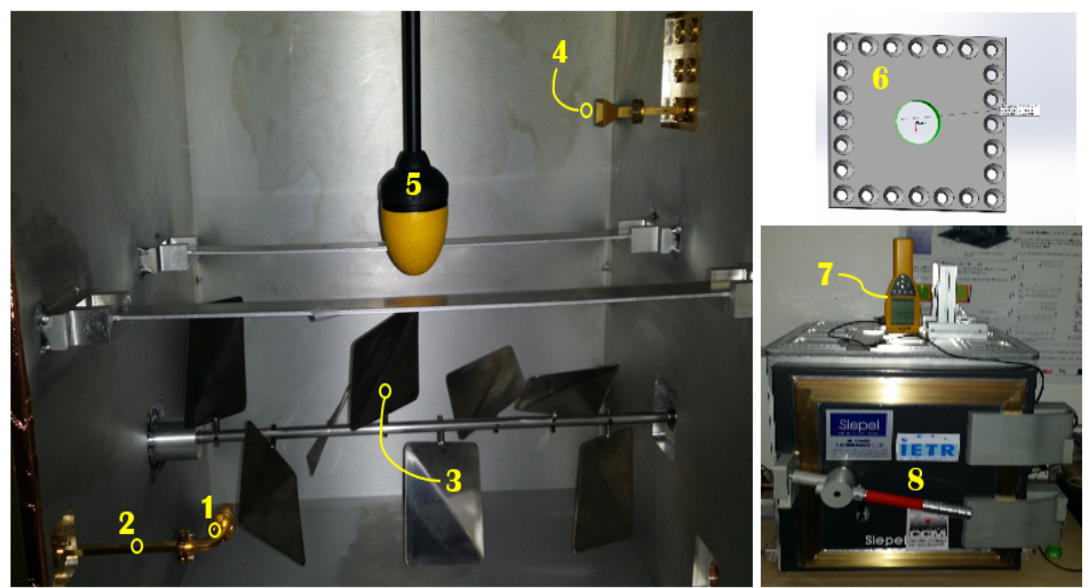

Fig. 3 The proposed measurement setup in the empty RC (setup 1 in Fig. 2): (1) openended waveguide (Tx); (2) waveguide from mm-wave source; (3) stirrer; (4) horn antenna connected to the power meter; (5) Narda EF6092 probe; (6) designed interface for the Narda probe; (7) Narda NBM-550 broadband field meter; (8) reverberation chamber (RC).

\subsubsection{Setup 2: on-phantom calibration using the Rx antenna}

The goal is to calibrate the single-antenna exposure meter $(\mathrm{Rx})$ on a tissueequivalent phantom in the $\mathrm{RC}$ in the $60 \mathrm{GHz}$ band. Figure 4 demonstrates the measurement setup. A ZVA 67 (Rohde\&Schwarz, Munich, Germany) vector network analyzer (VNA) is used to feed a standard open-ended waveguide $(0 \mathrm{dBm})$ and to measure the S-parameters. The $\mathrm{Rx}$ is fed with a $\mathrm{V}$-connector that has a loss of $0.8 \mathrm{~dB}$ [29]. The on-body calibration consists of three types of measurements: on-phantom (step 1 in Fig. 3), far from the phantom (step 2) and in free space (step 3, in the empty chamber).

For on-phantom measurements a skin-equivalent phantom [30] is used. The phantom mimics the dielectric properties of human skin in the range of 55$65 \mathrm{GHz}$. The dimensions of the phantom are $15 \times 15 \times 1 \mathrm{~cm}^{3}$ and its composition is presented in Table 1 . The complex permittivity of the phantom is measured using an open-ended coaxial probe (DAK 1.2E, Speag, Switzerland) and is equal to $7.4-j 11.4$ at $60 \mathrm{GHz}$. This is very close to the complex permittivity of dry skin at $60 \mathrm{GHz}$ that is $7.98-j 10.90$ [31]. Figure 5 shows a grid composed of nine antenna locations (denoted as $i$ ) on the phantom. The distance between locations are about $8 \lambda$, well above the spatial correlation of fields in $\mathrm{RC}(\lambda / 2)$.

In the first step (Fig. 2: step 1), the $\mathrm{Rx}$ is placed at every location $i$ $(i=1: 9)$ of Fig. 5(a) and the scattering (S) parameters were measured for 100 positions of the stirrer in the range of 59.5 to $60.5 \mathrm{GHz}$ and for every positions of the Rx on the phantom.

The $Q$-factor of the chamber is calculated from the S-parameters [28]: 


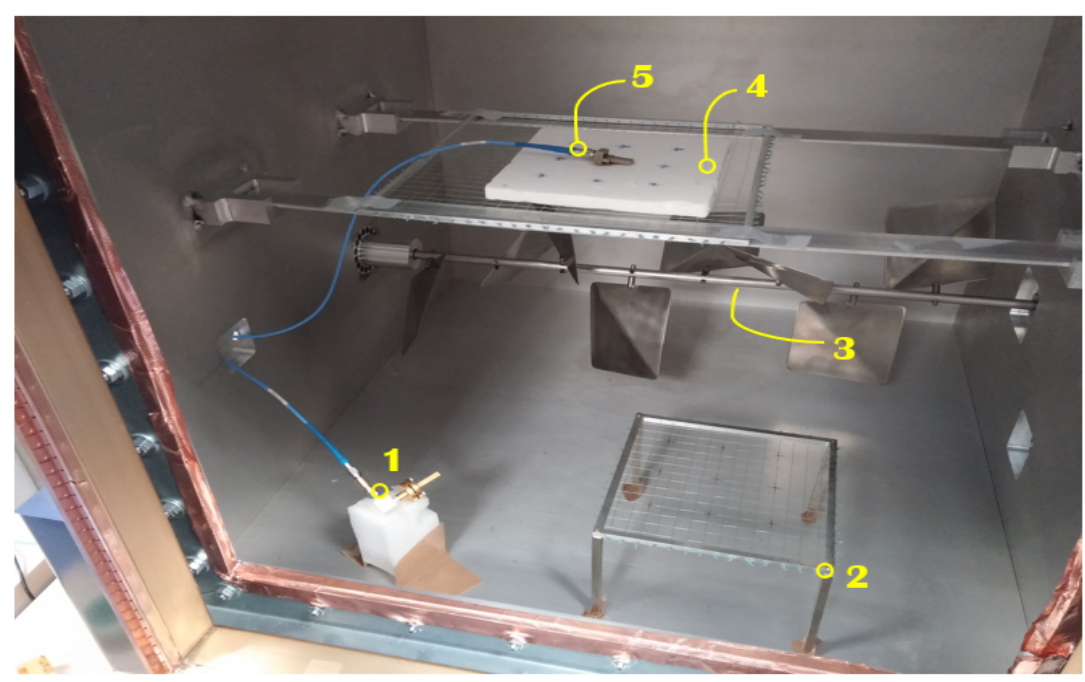

Fig. 4 The proposed measurement setup (setup 2 in Fig. 2) for on-body calibrations: (1) open-ended waveguide; (2) Rx holder for free space measurements either in loaded or empty RC; (3) stirrer; (4) skin phantom; (5) four-patch antenna array ( $\mathrm{Rx}$ ).

$$
Q(f)=\frac{16 \pi^{2} V f^{3}}{c^{3}} \frac{\left\langle P_{r, i}(f)\right\rangle}{P_{t}(f)}
$$

where $\mathrm{V}$ is the volume of the chamber, $f$ is the frequency, $c$ is the speed of light, $\left\langle P_{r, i}\right\rangle$ is the average received power on the $\mathrm{Rx}$ during the stirring process (at every location $i$ ) and $P_{t}$ is the transmitted power by the VNA. The relationship between the received power and the transmitted power in the $\mathrm{RC}$ can be expressed by the transmission factor $G[32,33]$ and is obtained from the measured S-parameters:

$$
G=\frac{\left\langle\left|S_{21}\right|^{2}-\left|\left\langle S_{21}\right\rangle\right|^{2}\right\rangle}{\left(1-\left|\left\langle S_{11}\right\rangle\right|^{2}\right)\left(1-\left|\left\langle S_{22}\right\rangle\right|^{2}\right) \eta_{e} \eta_{r}}
$$

where $\left|S_{21}\right|$ is the magnitude of forward transmission coefficient, $S_{11}$ and $S_{22}$ are the measured reflection coefficients of the transmitting and receiving antennas, respectively. Moreover, $\eta_{e}$ and $\eta_{r}$ denote the radiation efficiencies of the Tx and the $\mathrm{Rx}$, respectively. The notation \langle\rangle stands for an average over the stirrer's positions. It must be noted that the term $\left|\left\langle S_{21}\right\rangle\right|^{2}$ in the nominator is to take any direct or unstirred path during the measurements into account [33]. Considering (3) and (4), the Q-factor for every position of $i$ can be written as:

$$
Q=\frac{16 \pi^{2} V G}{\lambda^{3}}
$$


Table 1 Composition of the proposed human skin-phantom.

\begin{tabular}{ll}
\hline Ingredients & Mass (g) \\
\hline Water & 200 \\
Polyethylene & 40 \\
TX-151 & 4 \\
Agar & 3 \\
\hline
\end{tabular}

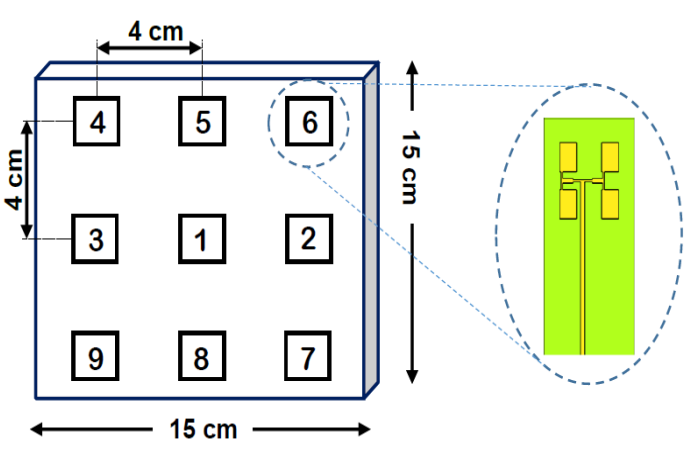

(a)

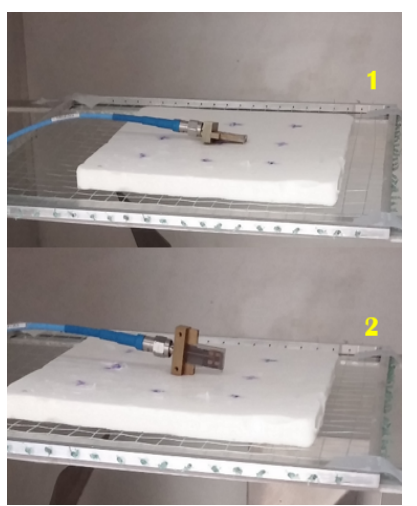

(b)

Fig. 5 The proposed locations and orientations of the antenna(s) on the skin phantom. (a) The geometry of the proposed antenna locations. The thickness of the phantom is $1 \mathrm{~cm}$. (b) The proposed orientations of the antenna(s) on the skin phantom: (1) default orientation of the nodes; (2) rotated antenna by 90 degrees.

where $\lambda$ is the wavelength. The Q-factor is averaged over nine positions of the Rx and the average IPD $\left\langle S_{i n c}\right\rangle$ on the phantom is calculated from the average Q-factor $\langle Q\rangle[26]$ :

$$
\left\langle S_{i n c}\right\rangle=\frac{\langle Q\rangle P_{t} \lambda}{2 \pi V}
$$

For $P_{t}$ the loss in the cable connecting the VNA to the Tx is considered $\left(P_{t}=P_{V N A}+\right.$ loss $\left._{\text {cable }}\right)$. Although calibration of the exposure meter requires the IPD to be measured in presence and absence of the phantom (in empty $\mathrm{RC}$ ), the power density inside the RC is proportional to the loaded Q-factor and the input power [26]. Therefore, moving the phantom in the $\mathrm{RC}$ or out of the $\mathrm{RC}$ at mm-waves affects the Q-factor significantly (loaded vs. empty) [26, 28]. Due to its large size with respect to the wavelength any modification of the phantom position in the $\mathrm{RC}$ induces a random modification of the plane wave spectrum and might introduce coupling effects with $\mathrm{Rx}$ antennas. Removing the phantom from the RC decreases the losses in the RC and yield an increase of the Q-factor. Should no direct coupling occurred in presence of the phantom, $\mathrm{Q}$-factor variation is theoretically retrieved from phantom properties. 
In the second step (see Fig. 2), the phantom is moved at least $50 \lambda$ away from the $\mathrm{Rx}$, and calibration measurements are repeated for the Rx antenna (with the phantom far from the $\mathrm{Rx}$ ) exactly at the same (nine) locations in the previous step. This is to emulate the free space conditions for the $\mathrm{Rx}$ in the loaded RC.

In the third step (Fig. 2), the phantom was removed out of the RC and measurements were repeated on the same locations of $\mathrm{Rx}$ and the IPD and Q-factor were determined for the empty RC.

The four-patch antenna array $(\mathrm{Rx})$ has a narrow beam width and a thick ground plane. Therefore, in order to study the effect of lossy phantom on its radiation pattern and thus on the response of the exposure meter, the $\mathrm{Rx}$ is rotated $90^{\circ}$ (see Fig. 5(b)) and the calibration measurements are repeated for three locations of $\mathrm{Rx}$ on the phantom $(i=1,2,3)$ and $\left\langle S_{\text {inc }}\right\rangle$ and $\langle Q\rangle$ are calculated.

\subsubsection{Measured response of the $m m$-PEM}

The response $R$ of the proposed exposure meter is defined as the ratio of the received power on the four-patch antenna array placed on the phantom $\left(P_{r, p h}\right)$ to the antenna placed in free space $\left(P_{r, \text { free }}\right)$, meaning that the $\mathrm{Rx}$ is far from the phantom or is in the empty RC:

$$
R=\frac{P_{r, p h}}{P_{r, \text { free }}}
$$

The received power $\left(P_{r}\right)$ on the antenna can be written as:

$$
P_{r}=\frac{\lambda^{2}}{8 \pi} \eta \frac{c}{z_{0}} S_{11}=A A \eta S_{11} \frac{c}{z_{0}}=S_{21}^{2} \times P_{i n}
$$

Using equations (7) and (8), $\mathrm{R}$ can be calculated from the ratio of the antenna apertures for the antenna on phantom and in free space. $A A$ is determined from the average Q-factor [33] in each measurement scenario:

$$
R=\frac{A A_{\text {ph }}}{A A_{\text {free }}}=\frac{\left\langle\left|S_{21, p h}\right|^{2}-\left|\left\langle S_{21, p h}\right\rangle\right|^{2}\right\rangle}{\left\langle\left|S_{21, \text { free }}\right|^{2}-\left|\left\langle S_{21, \text { free }}\right\rangle\right|^{2}\right\rangle}
$$

where $A A_{p h}$ is the antenna aperture for the $\mathrm{Rx}$ on the phantom and $A A_{\text {free }}$ is the antenna aperture for the $\mathrm{Rx}$ in absence of the phantom (far from the phantom: loaded RC or no phantom i.e. empty $\mathrm{RC})$.

Ideally, we target the response $R$ of the mm-PEM to be equal to 1 , so the mm-PEM measures the same IPD in free space and on the body (phantom). Our hypothesis is that this can be achieved by combining nine elements. 


\subsubsection{Average absorption cross section (AACS) of the phantom}

The average absorption cross section of the phantom (AACS) placed in the $\mathrm{RC}$ is determined from the Q-factors of the loaded $\mathrm{RC}$ ( $\mathrm{Rx}$ far from the phantom) and the empty (unloaded) RC and are denoted as $Q_{\text {far }}$ and $Q_{\text {empty }}$, respectively [32]:

$$
A A C S=\frac{2 \pi V}{\lambda}\left(\frac{1}{Q_{\text {far }}}-\frac{1}{Q_{\text {empty }}}\right)
$$

\subsection{Numerical Modeling}

The goal is to numerically determine the response of the exposure meter around $60 \mathrm{GHz}$ (in the range of $59.5 \mathrm{GHz}$ to $60.5 \mathrm{GHz}$ ) in diffuse environments. Figure 6 illustrates the proposed numerical model for the mm-wave exposure meter. The finite-difference time-domain (FDTD) simulation platform, Sim4Life [34], is used. The penetration depth of mm-waves into the body is very shallow and is limited to $0.48 \mathrm{~mm}$ at $60 \mathrm{GHz}$ [35]. Therefore, a homogenous model of human skin is employed for simulations. The dimensions and the dielectric properties of the numerical skin model are considered as $15 \times 15 \times 1 \mathrm{~cm}^{3}[36]$ and dry skin at $60 \mathrm{GHz}\left(\epsilon_{r}=7.97, \sigma=36.39 \mathrm{~S} / \mathrm{m}\right)[31]$, respectively. The antenna is placed at $5.6 \mathrm{~mm}$ from the surface of the skin model due to the geometry of the connector (see Fig. 6). The absorbing boundary conditions are set to uniaxial perfectly matched layers with a thickness of 7 layers. The skin model is resolved by a non-uniform mesh with maximum grid step of $100 \mu \mathrm{m}$. The simulation time is set to 50 periods to reach a steady state convergence. The entire geometry of the skin model and antenna is meshed by $197 \times 10^{6}$ voxels.
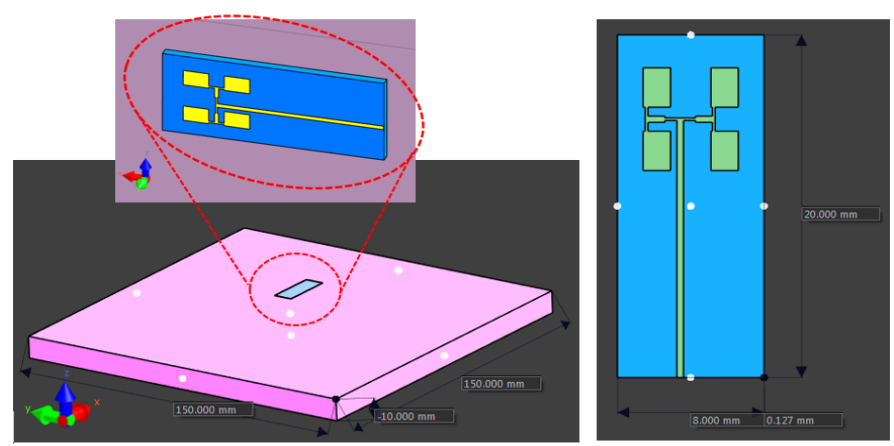

Fig. 6 The proposed model for numerical simulations. Small circle: location and orientation of the antenna on the phantom; large circle: the antenna is rotated by $90^{\circ}$. 


\subsubsection{Simulated response of the $m m-P E M$}

The simulated response of the mm-PEM is determined for five frequencies $(59.5,59.8,60,60.2$ and $60.5 \mathrm{GHz})$ as follows. First, a simulation is performed using the antenna in free space. Second, an on-body simulation is performed, and thus the antenna is placed on the center and at $5.6 \mathrm{~mm}$ from the surface of the skin model because of the connector size (see Fig. 6). Since the stirrer and the real $\mathrm{RC}$ are not modeled and the structure of the phantom is not symmetric, changing the antenna's location on the model will not affect the results. Therefore, a single simulation would suffice. The simulated response $R_{\text {sim }}$ is defined as the ratio of the average received power on the skin $P_{r, s i m}^{\text {skin }}$ to the average received power in free space $P_{r, s i m}^{f r e e}$ :

$$
R_{s i m}=\frac{P_{r, s i m}^{s k i n}}{P_{r, s i m}^{f r e e}}
$$

The received power $P_{r, \text { sim }}$ on an antenna can be determined from its antenna aperture (AA) [37]:

$$
P_{r, s i m}=S_{i n c} \times A A(\varphi, \theta)
$$

The $A A(\varphi, \theta)$ is obtained from the simulated directive gain $D(\varphi, \theta)$ of the antenna as:

$$
A A(\varphi, \theta)=\eta_{\text {rad }}\left(1-\left|S_{11}\right|^{2}\right) D(\varphi, \theta) \frac{\lambda^{2}}{4 \pi}
$$

where $\eta_{\text {rad }}$ is the radiation efficiency, $\left|S_{11}\right|$ is the antenna's power reflection coefficient and $\lambda$ is the wavelength. $A A(\varphi, \theta)$ can be determined for two orthogonal polarizations on the incident electric fields: $\theta$ and $\varphi$. These are polarizations parallel to the unity vectors $\overline{1}_{\theta}$ and $\overline{1}_{\phi}$. Using $A A(\varphi, \theta)$, the $P_{r, s i m}$ can be determined in diffuse fields. For multiple plane waves incident on the antenna, $P_{r, s i m}$ is not necessarily equal to the sum of the incident powers induced by each single plane wave, since the incident plane waves can interfere with each other. Therefore, the $P_{r, s i m}$ is calculated as a function of the incident electric fields (the sum of the induced voltages on the antenna) $[9,13]$. The phase, polarization, polar $(\theta)$ and azimuth $(\varphi)$ angles of the plane waves are assumed to have a uniform distribution (from which 1000 samples are drawn for each parameter) in the range of $[0, \pi]$ ( $\theta$ and polarization) and $[0,2 \pi](\varphi$ and phase) [23]. This results in a distribution for $P_{r, s i m}$.

\section{Results and Discussion}

Section 3.1 presents the measured $S_{i n c}$ by the mm-PEM, horn antenna and the isotropic probe in the empty RC. The Q-factor and the $S_{i n c}$ are calculated for the mm-PEM (Rx) on the phantom, far from the phantom and the rotated 
$\mathrm{Rx}$ on the phantom (Section 3.2). Next, the response of the mm-PEM is calculated (see Section 3.3). In Section 3.4, the AACS of the phantom is presented. Section 3.5 discusses the measurement uncertainties.

\subsection{Power density in unloaded (empty) RC}

Figure 7 shows the IPD $S_{\text {inc }}$ measured in the RC using the Narda probe, the horn antenna (eq 1) and the average over nine Rx elements (eq. 6). All the measurements are averaged over 100 positions of the stirrer. The results are normalized to an input power of $1 \mathrm{~W}$. The Narda probe measured the highest $S_{\text {inc }}$ compared to the horn antenna and the array antenna. At $60 \mathrm{GHz}$, the measured $S_{i n c}$ by the array antenna is $8.6 \mathrm{~W} \cdot \mathrm{m}^{-2}$ lower than the measured one by the horn antenna. The measured $S_{i n c}($ at $60 \mathrm{GHz}$ ) by the Narda probe is $136 \mathrm{~W} . \mathrm{m}^{-2}$ and $42.4 \mathrm{~W} . \mathrm{m}^{-2}$ larger than the measurement of the four-patch antenna array and the horn antenna, respectively. The difference for the two antennas is due to their different antenna apertures (AA), which depend on the directive gain and the antenna efficiency as well as the polarization (tri-axial versus linear). At $60 \mathrm{GHz}$, the standard deviations of the mean values are 53.4, 79.1 and $4.2 W \cdot m^{-2}$ for the Narda probe, horn antenna and the four-patch antenna array, respectively. Since it is not possible to place the Narda probe on the nine locations on the phantom to measure the $S_{\text {inc }}$, the measured values by the four-patch antenna array are used for calibration.

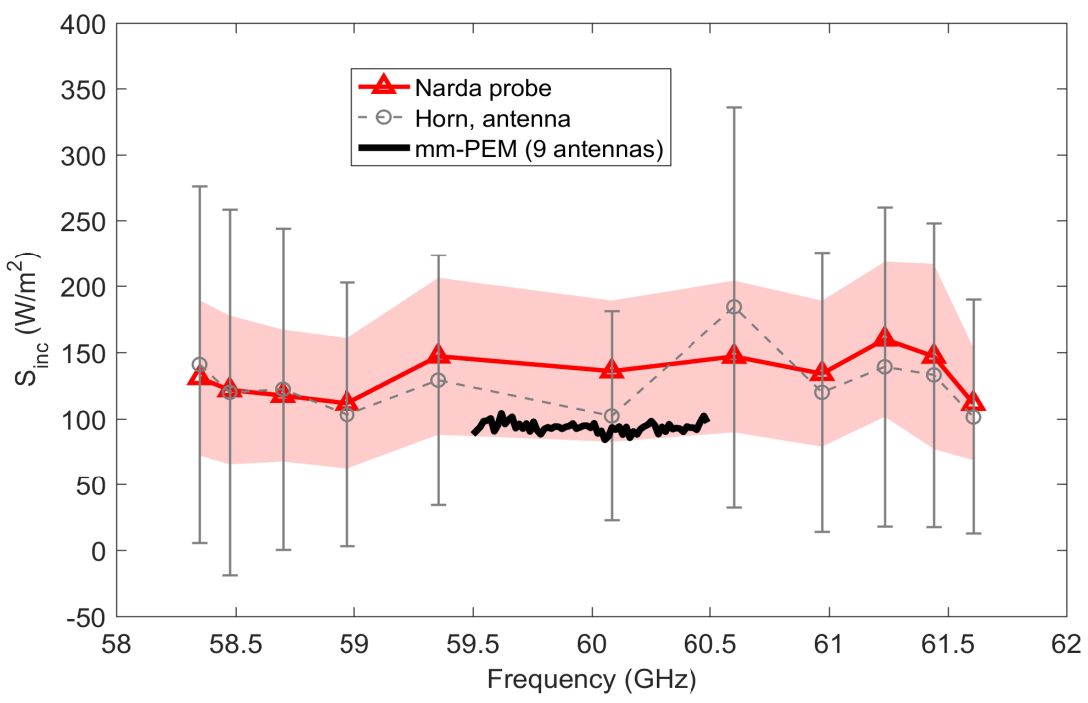

Fig. 7 The measured incident power density $\left(S_{\text {inc }}\right)$ in unloaded (empty) chamber. The results are normalized to an input power of $1 \mathrm{~W}$. All the values are averaged over 100 positions of the stirrer. Bars represent the standard deviations of the mean values for the Narda broadband probe and the horn antenna. 
3.2 Q-factor and incident power density: antenna array

Figure 8 depicts the measured quality factor of the empty and loaded RC averaged over nine antennas (see Fig. 5(a)) and 100 positions of the stirrer in the range of 59.5-60.5 GHz. At $60 \mathrm{GHz}$, the average quality factor $Q_{a v g}$ is estimated 26000 for the empty RC. Although loading the chamber with the phantom decreases $Q_{\text {avg }}$ to 7820 , these are sufficiently high values to ensure the correct functionality of the RC. The antenna on the phantom has a negligible influence on Q-factor which is only affected by the presence of the phantom itself. In other words, part of the radiation pattern of the four-patch antenna array is affected by the phantom (reflection and absorption). When the antenna is rotated $90^{\circ}$ on the phantom, a direct coupling path between antennas and the phantom results in higher losses and thus a lower Q-factor. Also the radiation pattern of the rotated antenna on phantom is more affected by the phantom. The Q-factor reduces from 7120 to 5990 when the antenna on the phantom is rotated $90^{\circ}$. The standard deviation of the average (over nineantenna elements and 100 positions of the stirrer) Q-factors are 2600, 705, 911 and 243 for the antennas in the empty chamber, far from the phantom (loaded $\mathrm{RC}$ ), on phantom and rotated on the phantom, respectively. This shows less variation of the Q-factor when nine-antenna elements are used.

Using (6), the $S_{i n c}$ averaged over nine antennas is calculated from $Q_{a v g}$. Figure 9 illustrates the measured $S_{i n c}$ for the antenna array in the empty and loaded chamber for an input power of $1 \mathrm{~W}$. The measured power density in the unloaded (empty) $\mathrm{RC}$ is $96.6 \mathrm{~W} \cdot \mathrm{m}^{-2}$ at $60 \mathrm{GHz}$ with a maximum measured value of $104 \mathrm{~W} \cdot \mathrm{m}^{-2}$ (at $59.6 \mathrm{GHz}$ ). The measured $S_{\text {inc }}$ for the loaded RC (antenna far from the phantom) is $12.1 \mathrm{~W} \cdot \mathrm{m}^{-2}$ at $60 \mathrm{GHz}$ which is at least 8.6 times lower when the $\mathrm{RC}$ is not loaded. Also rotating the antenna decreased $S_{\text {inc }}$ to $9.2 \mathrm{~W} \cdot \mathrm{m}^{-2}$ which is a $25 \%$ reduction in the received $S_{i n c}$ on the antenna due to the presence of the phantom.

\subsection{Response of the mm-PEM}

\subsubsection{Measured Response of mm-PEM (ratio of $A A$ )}

Figure 10 demonstrates the response (ratio of the antenna apertures) of the exposure meter for the antenna on the phantom and in absence of the phantom (loaded and the empty). It must be noted that the response is calculated for the average over nine locations of the antennas. The response $R$ of the exposure meter is 1.043 at $60 \mathrm{GHz}$ (Fig. 10) for the nine antennas on the phantom considering the free space $S_{i n c}$ is measured in the loaded chamber (antenna far from the phantom). Then the ideal value of $R=1$ is approached excellently by positioning the mm-PEM on the phantom. The response decreases to 0.27 (at $60 \mathrm{GHz}$ ) when the free space values in the empty $\mathrm{RC}$ are used. This is an underestimation by a factor of 3.5. Similarly, when the antenna is rotated, the 


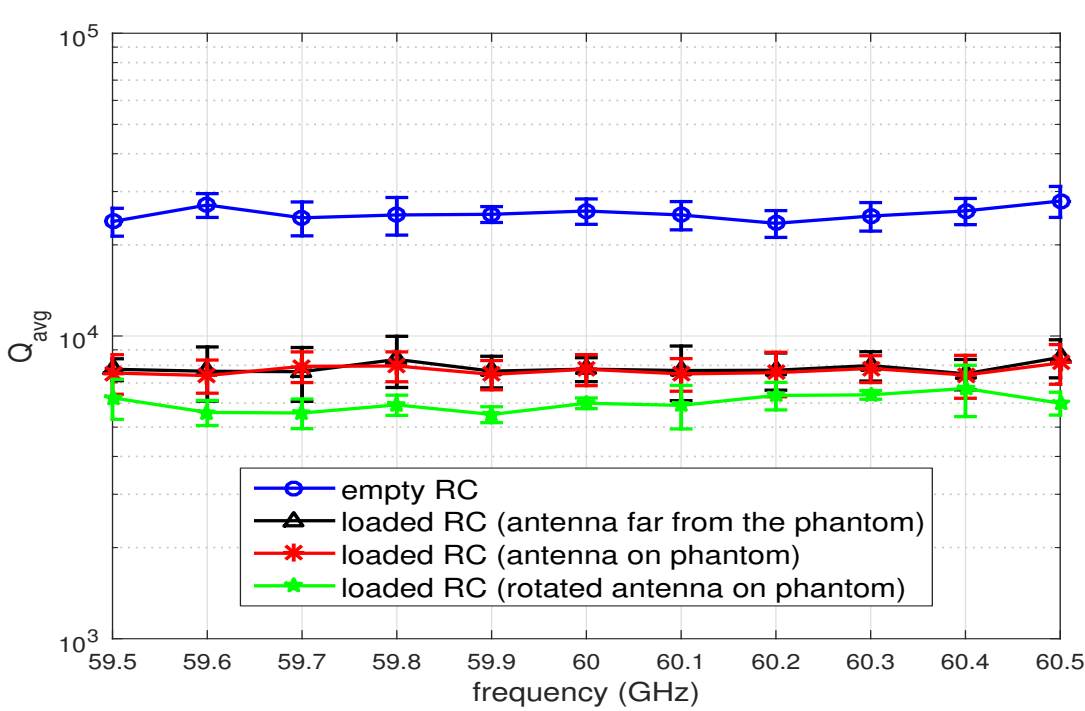

Fig. 8 The measured Q-factor of empty and loaded RC averaged over nine antennas and 100 positions of the stirrer. Error bars indicate the standard deviations.

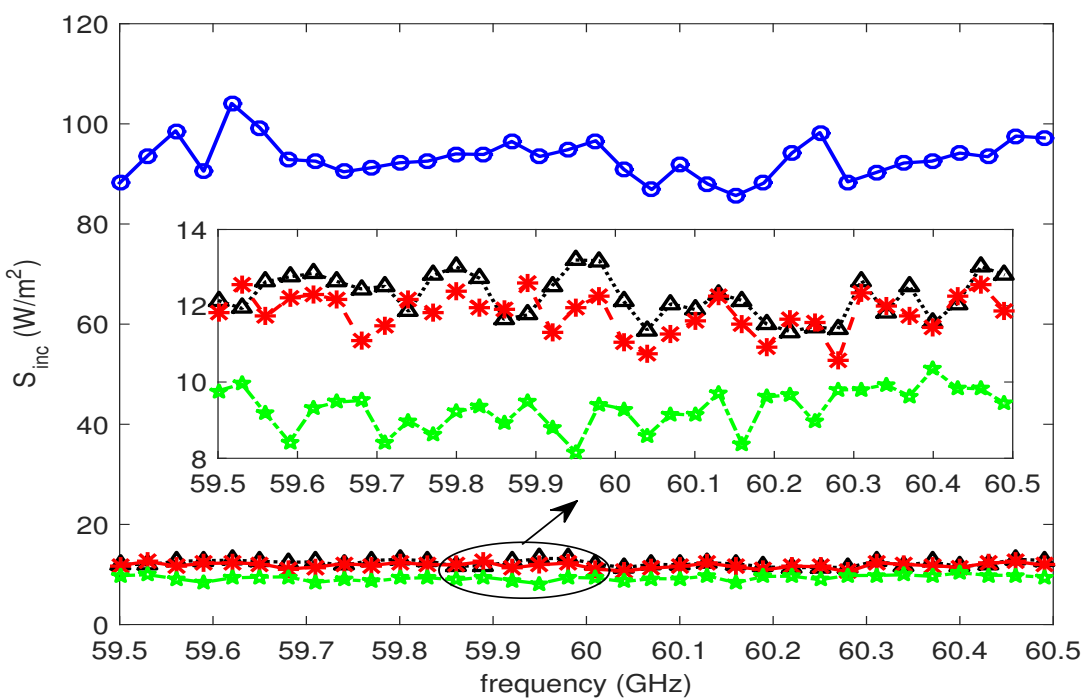

Fig. 9 The measured incident power density $S_{i n c}$ in the loaded and unloaded RC averaged over nine antennas and 100 positions of the stirrer. The measurements are normalized for an input power of $1 \mathrm{~W}$. Blue solid line: unloaded (empty) RC; black dotted line: loaded RC (antenna far from the phantom); red dashed line: loaded RC (antenna on phantom); green dashed-dotted line: loaded RC (rotated antenna on phantom).

response is 0.88 applying the free space values of $S_{i n c}$ in the loaded chamber, where the antenna is placed far from the phantom. 
When considering $S_{\text {inc }}$ values in the empty chamber the response for the rotated antenna on the phantom decrease from 0.88 to 0.21 which is a factor of 4. It can be concluded that at mm-waves, the calibration of the exposure meter has to be performed for the antenna(s) in the loaded chamber. Firstly, loading the RC affects its Q-factor. Figure 8 confirms this, in which, the Q-factor for the antenna on the phantom is very close to the Q-factor of the antenna far from the phantom. Secondly, the free space $S_{i n c}$ should be measured in the loaded RC, where the antenna is far from the phantom. Using nine antennas on the phantom, the $S_{\text {inc }}$ can be estimated with a ratio of $0.85(-0.66 \mathrm{~dB})$ to $1.13(0.55 \mathrm{~dB})$ w.r.t. the $S_{\text {inc }}$ in absence of the phantom (in this case far from the phantom, $50 \lambda$ ) in the range of 59.5 to $60.5 \mathrm{GHz}$.

Previously, the response of a mm-PEM at $60 \mathrm{GHz}$ has been reported in the range of 0.75 (on a skin phantom) to 0.8 (on body) in specular conditions [13]. The actual exposure (i.e. $S_{i n c}$ ) in a real environment should be in between both (specular and diffuse) estimates. The exact value in a real environment depends on the contribution of specular and diffuse fields.

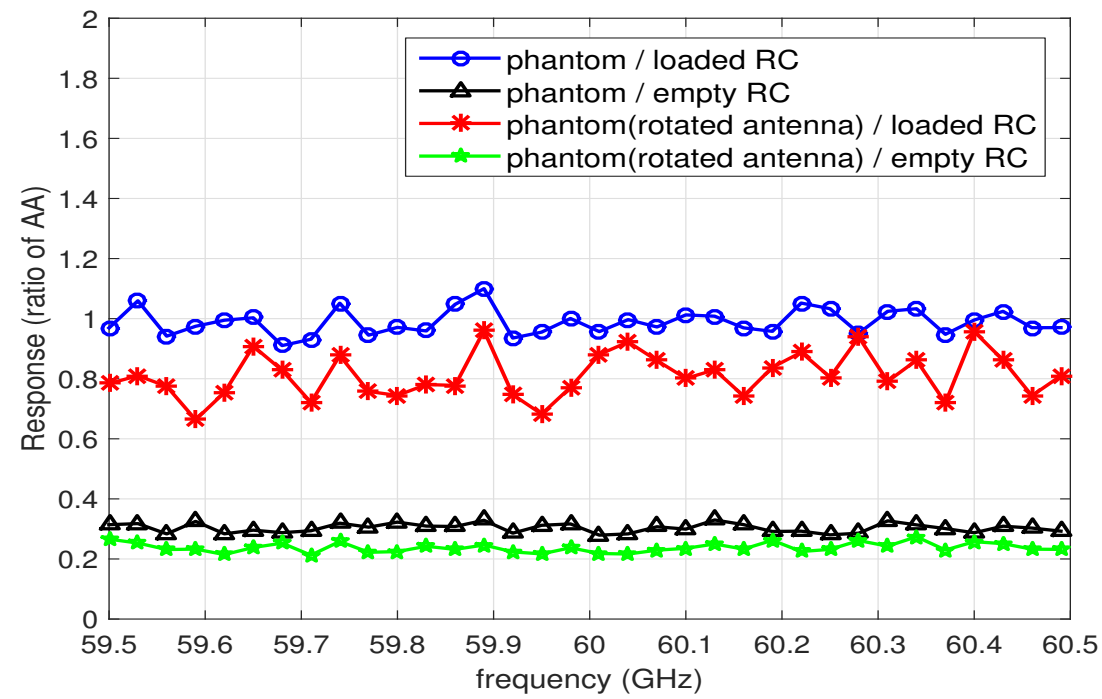

Fig. 10 Comparison of the response (ratio of antenna apertures) for two scenarios: empty $\mathrm{RC}$ and loaded RC.

The response of the mm-PEM is illustrated in Fig. 11 at $60 \mathrm{GHz}$ as a function of the number of $\mathrm{Rx}$ antennas. Using less than 3 antennas on the phantom leads to an overestimation of $S_{i n c}$ in the RC up to a factor of 1.25 $(0.96 \mathrm{~dB})$. Also using 4 nodes resulted in an underestimation of $0.9(-0.45 \mathrm{~dB})$. Increasing the number of antennas up to nine decreases the response of $\mathrm{mm}$ PEM to $1.043(0.17 \mathrm{~dB})$ at $60 \mathrm{GHz}$ and is very close to $1(0 \mathrm{~dB})$ which is the desired response of the mm-PEM. 


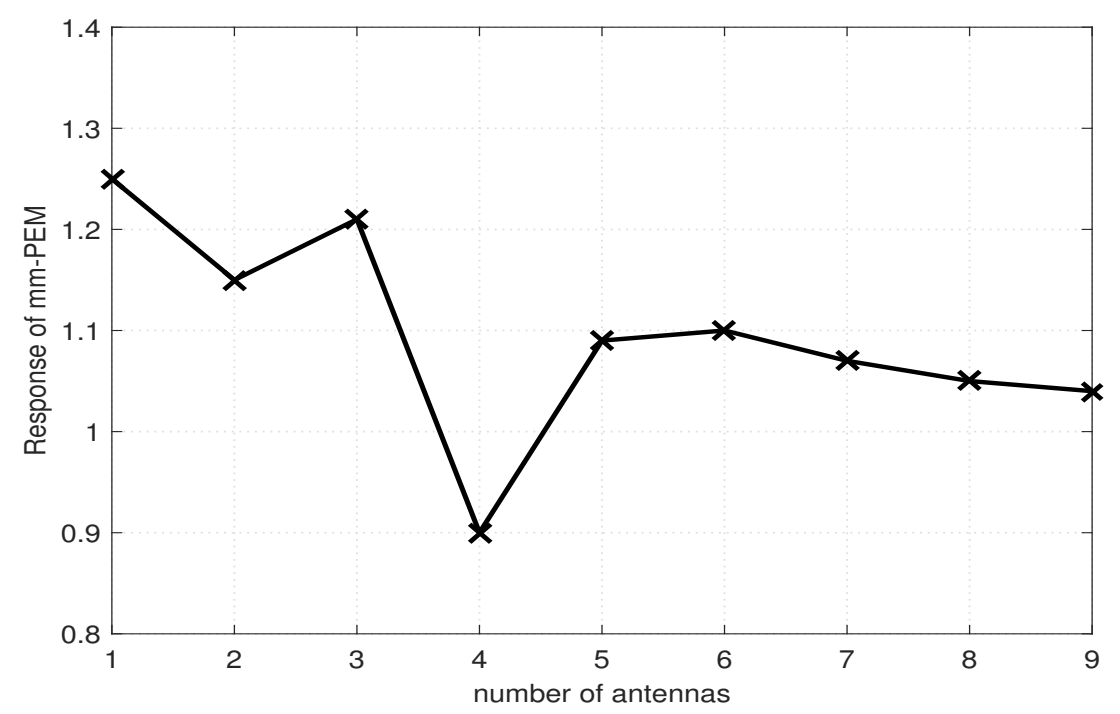

Fig. 11 The response of mm-PEM as a function of number of antennas (elements) at $60 \mathrm{GHz}$ using the free space values in the loaded $\mathrm{RC}$.

\subsubsection{Simulated Response of mm-PEM}

Figure 12 shows the simulated median response $R_{\text {sim }}$ (eq. 11) of the mm-PEM (single antenna) versus the measured response (ratio of AA, see Section 3.3.1) averaged over nine $\mathrm{Rx}$ antennas. The simulated response is in the range of 0.91 $(59.5 \mathrm{GHz})$ to $1.01(60.2 \mathrm{GHz})$. For the rotated $\mathrm{Rx}$ on the phantom, the $R_{\text {sim }}$ decreases to the range of $0.15(59.8 \mathrm{GHz})$ to $0.2(60 \mathrm{GHz})$. At $60 \mathrm{GHz}$ this is a factor of 4.6 reduction. The reason is the Rx's radiation along the skin's surface. The simulated $R_{\text {sim }}$ for the $\mathrm{Rx}$ on the phantom is in good agreement with the measured $R$ when the free space values in the loaded RC are used for calibration. But for the rotated $\mathrm{Rx}$, the simulated $R_{\text {sim }}$ is in good agreement with the measured $R$ when the free space in empty RC are used. It must be noticed that the simulation does not consider the Q-factor and is based on the received power on the antenna for two scenarios: in free space and on the phantom. Also since the real $\mathrm{RC}$ is not modeled, changing the location of the antenna on the phantom does not affect the results significantly.

\subsection{AACS}

Figure 13 depicts the average absorption cross section (AACS) of the phantom as a function of frequency. The measured AACS of the phantom is $225 \mathrm{~cm}^{2}$ at $60 \mathrm{GHz}$. Considering the dimensions of the phantom $(15 \mathrm{~cm} \times 15 \mathrm{~cm})$, this is an excellent agreement. A line is fitted to the measured data as a function of frequency. The line shows that increasing the frequency, does not affect the 


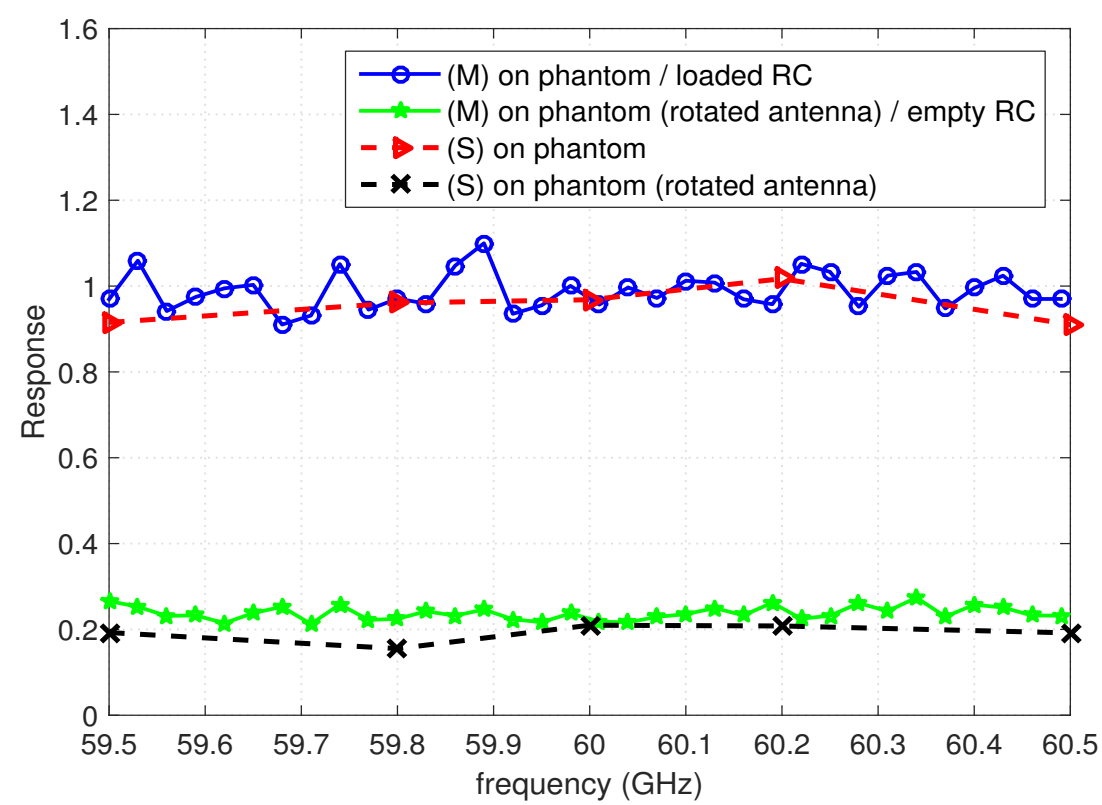

Fig. 12 Simulated median response $R_{\text {sim }}$ of (single antenna) mm-PEM vs. measured response (AA ratio) of the mm-PEM with nine antennas. (M) Measurement; (S) simulation. The measured $R$ values are those depicted in Fig. 10.

AACS significantly and the AACS is almost constant in the range of 59.4 to 60.6 GHz.

\subsection{Measurement uncertainty}

Using EF6092 probe in combination with the NBM-550 field meter causes a combined standard uncertainty of $1.86 \mathrm{~dB}$ (including linearity, frequency sensitivity, isotropic and thermal responses [38]) on the measurements in empty $\mathrm{RC}$ during the calibration measurements.

For the mm-PEM with nine antennas, the average $S_{i n c}$ is obtained from measurements of the Q-factor (see eq. 6). Therefore, the uncertainty of $S_{i n c}$ is related to the uncertainty of the measured Q-factor. The standard deviation $(\sigma)$ of $\mathrm{Q}$ is calculated as [39]:

$$
\sigma=\frac{1}{\sqrt{N \times P}}
$$

considering nine positions of the $\operatorname{Rx}(P=9)$ and 100 positions of the stirrer $(N=100)$, the standard deviation of the average power density is about $3.33 \%$. This results in a $95 \%$ confidence interval of $\pm 6.66 \%$ on the measured average $S_{\text {inc }}$ using the mm-PEM in the RC.

It should be noticed that since placement of the broadband field probe on the nine locations of the phantom was not possible in this measurement 


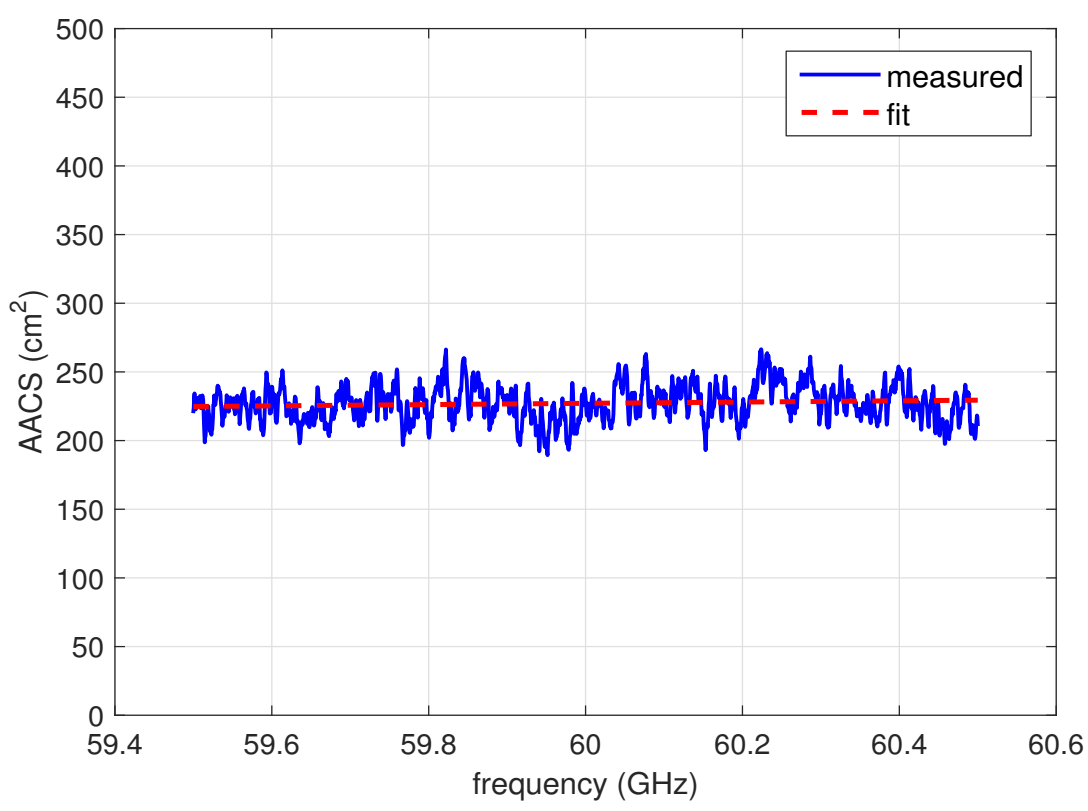

Fig. 13 The average absorption cross section of the phantom.

setup, the measurement uncertainty of the broadband probe and the proposed $\mathrm{mm}-\mathrm{PEM}$ are not comparable. In reality a prototype the mm-PEM uses a power detector thus, the contributions due to stability issues of feed power and sensor signal should be considered in the calibration which is part of the future research.

\section{Conclusion}

A mm-wave personal exposure meter (mm-PEM) is designed for assessment of personal exposure to $5 \mathrm{G}$ in indoor diffuse environments. The mm-PEM is calibrated on a skin-equivalent phantom in a reverberation chamber $(\mathrm{RC})$ in the range of 59.5 to $60.5 \mathrm{GHz}$. We showed that increasing the number of antennas up to nine, a response of 1.043 at $60 \mathrm{GHz}$ is obtained. This response is very close to 1 , which is the desired response of the mm-PEM so the mm-PEM can measure the IPD in free space but in the presence of human body. The response of the mm-PEM in diffuse fields is also determined in terms of numerical simulations using the FDTD. Good agreement between measurements and simulations is achieved. According to the results, we recommend to calibration the mm-PEM in the loaded RC. The average absorption cross section of the skin-phantom is determined as $225 \mathrm{~cm}^{2}$ from the measurements, which is in excellent agreement with the physical geometry of the phantom. Future research includes design of acquisition nodes (antenna and receiver electronics) for the mm-PEM to measure the IPD directly. Additionally, the calibrations 
will be performed on a cylindrical or spherical phantom to study the effect of body shadowing on the response of the designed mm-PEM and to design a distributed exposure meter for the mm-waves.

Acknowledgements This work was supported by the Research Foundation - Flanders (FWO-V) under grant agreement No G003415N. A.T. has received funding from the European Union's Horizon 2020 research and innovation programme under the Marie SkłodowskaCurie grant agreement No 665501 with the FWO. A.T. is an FWO [PEGASUS] ${ }^{2}$ Marie Skłodowska-Curie Fellow.

\section{References}

1. A. Osseiran, F. Boccardi, V. Braun, K. Kusume, P. Marsch, M. Maternia, O. Queseth, M. Schellmann, H. Schotten, H. Taoka, H. Tullberg, M.A. Uusitalo, B. Timus, M. Fallgren, Scenarios for $5 \mathrm{G}$ mobile and wireless communications: the vision of the metis project, IEEE Communications Magazine 52(5), 26 (2014). DOI 10.1109/MCOM.2014. 6815890

2. D. Andreuccetti, R. Fossi, C. Petrucci. The 5 G infrastructure public private partnership: the next generation of communication networks and services. URL https://5g-ppp. eu/wp-content/uploads/2015/02/5G-Vision-Brochure-v1.pdf. Accessed on (Feb. 01, 2018)

3. Z. Pi, F. Khan, An introduction to millimeter-wave mobile broadband systems, IEEE Communications Magazine 49(6), 101 (2011). DOI 10.1109/MCOM.2011.5783993

4. T.S. Rappaport, S. Sun, R. Mayzus, H. Zhao, Y. Azar, K. Wang, G.N. Wong, J.K. Schulz, M. Samimi, F. Gutierrez, Millimeter wave mobile communications for $5 \mathrm{G}$ cellular: It will work!, IEEE Access 1, 335 (2013). DOI 10.1109/ACCESS.2013.2260813

5. International Commission on Non-ionizing Radiation Protection (ICNIRP), Guidelines for limiting exposure to time-varying electric, magnetic, and electromagnetic fields (up to $300 \mathrm{GHz}$ ), Health Phys. 74(4), 494 (1998)

6. J.F. Bolte, G. van der Zande, J. Kamer, Calibration and uncertainties in personal exposure measurements of radiofrequency electromagnetic fields, Bioelectromagnetics 32(8), $652(2011)$

7. J. Blas, F.A. Lago, P. Fernández, R.M. Lorenzo, E.J. Abril, Potential exposure assessment errors associated with body-worn RF dosimeters, Bioelectromagnetics 28(7), 573 (2007)

8. A. Thielens, S. Agneessens, H. De Clercq, J. Lecoutere, L. Verloock, E. Tanghe, S. Aerts, R. Puers, H. Rogier, L. Martens, W. Joseph, On-body calibration and measurements using a personal, distributed exposimeter for wireless fidelity, Health Phys 108(4), 407 (2015)

9. R. Aminzadeh, A. Thielens, A. Bamba, L. Kone, D.P. Gaillot, M. Lienard, L. Martens, W. Joseph, On-body calibration and measurements using personal radiofrequency exposimeters in indoor diffuse and specular environments, Bioelectromagnetics 37(5), 298 (2016)

10. J.F. Bolte, Lessons learnt on biases and uncertainties in personal exposure measurement surveys of radiofrequency electromagnetic fields with exposimeters, Environment International 94(Supplement C), 724 (2016). DOI 10.1016/j.envint.2016.06.023

11. R. Aminzadeh, A. Thielens, S. Agneessens, P. Van Torre, M. Van den Bossche, S. Dongus, M. Eeftens, A. Huss, R. Vermeulen, R. de Seze, P. Mazet, E. Cardis, H. Rogier, M. Röösli, L. Martens, W. Joseph, A multi-band body-worn distributed radiofrequency exposure meter: Design, on-body calibration and study of body morphology, Sensors 18(272) (2018). DOI 10.3390/s18010272

12. A. Thielens, L. Martens, W. Joseph, Can body-worn devices be used for measuring personal exposure to mm waves?, Bioelectromagnetics 38(3), 239 (2017). DOI 10.1002/ bem. 22036 
13. R. Aminzadeh, A. Thielens, H. Li, C. Leduc, M. Zhadobov, G. Torfs, J. Bauwelinck, L. Martens, W. Joseph, Personal Exposimeter for Radiation Assessment in Real Environments in the 60-GHz Band, Radiation Protection Dosimetry 176(3), 316 (2017). DOI $10.1093 / \mathrm{rpd} / \mathrm{ncx} 012$

14. B. Thors, D. Colombi, Z. Ying, T. Bolin, C. Törnevik, Exposure to rf emf from array antennas in 5G mobile communication equipment, IEEE Access 4, 7469 (2016). DOI 10.1109/ACCESS.2016.2601145

15. A.R. Guraliuc, M. Zhadobov, R. Sauleau, L. Marnat, L. Dussopt, Near-field user exposure in forthcoming $5 \mathrm{~g}$ scenarios in the $60 \mathrm{GHz}$ band, IEEE Transactions on Antennas and Propagation 65(12), 6606 (2017). DOI 10.1109/TAP.2017.2754473

16. M. Kojima, Y. Suzuki, C.Y. Tsai, K. Sasaki, K. Wake, S. Watanabe, M. Taki, Y. Kamimura, A. Hirata, K. Sasaki, H. Sasaki, Characteristics of ocular temperature elevations after exposure to quasi- and millimeter waves (18-40 GHz), Journal of Infrared, Millimeter, and Terahertz Waves 36(4), 390 (2015). DOI 10.1007/s10762-014-0142-4

17. J.X. Zhao, H.K. Zhang, D.G. Liu, D.Q. Lu, Millimeter-wave irradiation dosimetry for cells cultured in petri dish, International Journal of Infrared and Millimeter Waves 25(10), 1439 (2004). DOI 10.1023/B:IJIM.0000047436.12820.50

18. M. Zhadobov, R. Sauleau, R. Augustine, C. Le Quément, Y. Le Dréan, D. Thouroude, Near-field dosimetry for in vitro exposure of human cells at $60 \mathrm{GHz}$, Bioelectromagnetics 33(1), 55 (2012). DOI 10.1002/bem.20685

19. M. Zhadobov. Antenna/human body interactions in the $60 \mathrm{GHz}$ band: state of knowledge and recent advances (2016). DOI 10.1049/PBTE065E_ch5. URL http: //digital-library.theiet.org/content/books/10.1049/pbte065e_ch5

20. Z.M. C., A.S. I., F.K. R., B. Quirino, Tissue models for rf exposure evaluation at frequencies above $6 \mathrm{GHz}$, Bioelectromagnetics 39(3), 173 (2018). DOI 10.1002/bem. 22110

21. T. Wu, T.S. Rappaport, C.M. Collins, Safe for generations to come: Considerations of safety for millimeter waves in wireless communications, IEEE Microwave Magazine 16(2), 65 (2015). DOI 10.1109/MMM.2014.2377587

22. C. Aliaga. How is the time of women and men distributed in europe. statistics in focus, population and social conditions. issn 1024-4352, ks-nk-06-004-en-n, eurostat Luxembourg (2006). URL http://www.unece.org/fileadmin/DAM/stats/gender/timeuse/ DataReports/How_is_the_time_of_Women_and_Men.pdf. Accessed on (Feb. 01, 2018)

23. J.B. Andersen, J.O. Nielsen, G.F. Pedersen, G. Bauch, J.M. Herdin, Room electromagnetics, IEEE Antennas and Propagation Magazine 49(2), 27 (2007). DOI 10.1109/MAP.2007.376642

24. J. Poutanen, J. Salmi, K. Haneda, V.M. Kolmonen, P. Vainikainen, Angular and shadowing characteristics of dense multipath components in indoor radio channels, IEEE Transactions on Antennas and Propagation 59(1), 245 (2011). DOI 10.1109/TAP.2010. 2090474

25. A.K. Fall, P. Besnier, C. Lemoine, M. Zhadobov, R. Sauleau, Design and experimental validation of a mode-stirred reverberation chamber at millimeter waves, IEEE Transactions on Electromagnetic Compatibility 57(1), 12 (2015). DOI 10.1109/TEMC.2014. 2356712

26. A.K. Fall, P. Besnier, C. Lemoine, M. Zhadobov, R. Sauleau, Experimental dosimetry in a mode-stirred reverberation chamber in the $60-\mathrm{GHz}$ band, IEEE Transactions on Electromagnetic Compatibility 58(4), 981 (2016). DOI 10.1109/TEMC.2016.2550803

27. N. Chahat, M. Zhadobov, L.L. Coq, S.I. Alekseev, R. Sauleau, Characterization of the interactions between a $60-\mathrm{GHz}$ antenna and the human body in an off-body scenario, IEEE Transactions on Antennas and Propagation 60(12), 5958 (2012). DOI 10.1109/ TAP.2012.2211326

28. D.A. Hill, M.T. Ma, A.R. Ondrejka, B.F. Riddle, M.L. Crawford, R.T. Johnk, Aperture excitation of electrically large, lossy cavities, IEEE Transactions on Electromagnetic Compatibility 36(3), 169 (1994). DOI 10.1109/15.305461

29. N. Chahat, M. Zhadobov, L.L. Coq, R. Sauleau, Wearable endfire textile antenna for on-body communications at $60 \mathrm{GHz}$, IEEE Antennas and Wireless Propagation Letters 11, 799 (2012). DOI 10.1109/LAWP.2012.2207698 
30. N. Chahat, M. Zhadobov, R. Sauleau, Broadband tissue-equivalent phantom for ban applications at millimeter waves, IEEE Transactions on Microwave Theory and Techniques 60(7), 2259 (2012). DOI 10.1109/TMTT.2012.2195196

31. D. Andreuccetti, R. Fossi, C. Petrucci. An internet resource for the calculation of the dielectric properties of body tissues in the frequency range $10 \mathrm{~Hz}-100 \mathrm{GHz}$ (1997). URL http://niremf.ifac.cnr.it/tissprop/. IFAC-CNR, Florence (Italy), 1997. Based on data published by C.Gabriel et al. in 1996

32. D. Senić, C. Holloway, J. Ladbury, G. Koepke, A. Šarolić, in 2014 International Symposium on Electromagnetic Compatibility (2014), pp. 962-967. DOI 10.1109/EMCEurope. 2014.6931042

33. P. Besnier, C. Lemoine, J. Sol, in 2015 IEEE International Symposium on Electromagnetic Compatibility (EMC) (2015), pp. 1223-1227. DOI 10.1109/ISEMC.2015.7256344

34. Schmid \& Partner Engineering AG (SPEAG), Sim4Life Reference Manual (2017). Http://www.zurichmedtech.com/sim4life/

35. M. Zhadobov, N. Chahat, R. Sauleau, C. Le-Quement, Y.L. Drean, Millimeter-wave interactions with the human body: state of knowledge and recent advances, Int J Microw Wirel T. 3(02), 237 (2011)

36. R. Aminzadeh, M. Saviz, A.A. Shishegar, in 2014 22nd Iranian Conference on Electrical Engineering (ICEE) (2014), pp. 1657-1661. DOI 10.1109/IranianCEE.2014.6999804

37. C.A. Balanis, Antenna Theory: Analysis and Design (Wiley-Interscience, 2005)

38. Narda Safety Test Solutions. Electric and Magnetic Field Measurement, NBM Series Probes., EF6092: Sensitive field probe for all types of high frequency application.100 $\mathrm{MHz}-60 \mathrm{GHz}$ (2016). NBM-Probes: DataSheet

39. J.G. Kostas, B. Boverie, Statistical model for a mode-stirred chamber, IEEE Transactions on Electromagnetic Compatibility 33(4), 366 (1991). DOI 10.1109/15.99120 